\title{
Enfermeiro Gestor de Caso na promoção da prevenção da Violência Doméstica - Revisão Integrativa
}

\author{
Ana Paula Reis Carmona ${ }^{1}$, Maria da Graça Quaresma ${ }^{1}$ e Pedro Lucas ${ }^{1}$ \\ ${ }^{1}$ Escola Superior de Enfermagem de Lisboa (ESEL), Portugal | anapcarmona@gmail.com; \\ graca.quaresma@chlc.min-saude.pt; prlucas@esel.pt | https://orcid.org/0000-0003-0301- \\ 2261; https://orcid.org/0000-0001-8567-0983; https://orcid.org/0000-0002-4816-0762
}

\begin{abstract}
Resumo: A Violência doméstica é um importante problema de Saúde Pública devido à sua magnitude, frequência e repercussões para a saúde dos indivíduos, famílias, grupos e comunidades. E um fenómeno multifactorial definido pela Organização Mundial de Saúde (OMS) (Taft et al., 2012) Introdução: Os Enfermeiros detêm um importante papel nas equipas de saúde, tanto no reconhecimento da situação de violência como na resposta de prestação de cuidados às vítimas e suas famílias pelo que a Liderança é uma aptidão extraordinariamente necessária e exigida aos enfermeiros para influenciar as suas equipas a prestar cuidados de enfermagem que respondam às expectativas dos utentes (Amestoy et al., 2014); Objetivos: Identificar a evidência científica sobre a intervenção do enfermeiro gestor de caso, na promoção da prevenção da Violência Doméstica. Métodos: Revisão Integrativa da Literatura de estudos indexados nas bases de dados MEDLINE (PubMed/Medline), CINAHL, Cochrane, e em outras bases de dados da plataforma EBSCOHost (LILACS, SCOPUS e Web of Science), e ainda uma pesquisa nas referências bibliográficas dos artigos selecionados. Os estudos selecionados são referentes ao período entre 2010 e 2020; Resultados: Foram incluídos 11 artigos sobre implementação de programas de deteção e prevenção da Violência Doméstica com resultados para as vítimas, para os enfermeiros, para os enfermeiros gestores e para a eficiência das organizações, em Cuidados de Saúde Primários e em Hospitais. Conclusões: A Implementação de Modelos de Gestão de Caso melhora a resposta às vítimas de violência, com consequências na qualidade dos cuidados prestados, com aumento de resultados na prevenção. Não foram encontrados estudos relacionando o enfermeiro gestor de caso para a violência doméstica, em Cuidados de Saúde Primários.
\end{abstract}

Palavras-chave: Enfermagem; Administração de Caso; Violência Doméstica; Cuidados de Saúde Primários; Revisão.

Nurse Case Manager in the Promotion and Prevention of Domestic Violence - Integrative Review

Abstract: Domestic violence is an important public health problem due to its magnitude, frequency and repercussions for the health of individuals, families, groups and communities. It is a multifactorial phenomenon defined by the World Health Organization (WHO) (Taft et al., 2012) Introduction (Nurses play an important role in health teams, both in recognizing the situation of violence and in responding to the provision of care to victims and their families, for which Leadership is an extraordinarily necessary and required skill for nurses to influence their teams to provide nursing care that responds to users' expectations (Amestoy et al., 2014); Goals Identify the scientific evidence on the intervention of the case manager nurse in the promotion of the prevention of Domestic Violence; Methods Integrative Literature Review of studies indexed in the MEDLINE databases (PubMed / Medline), CINAHL, Cochrane, and in other databases of the EBSCOHost platform (LILACS, SCOPUS and Web of Science), and also a search in the bibliographic references of the articles selected. The selected studies are for the period between 2010 and 2020; Results Eleven articles were included on the implementation of Domestic Violence detection and prevention programs with results for victims, for nurses, for nurse managers and for the efficiency of organizations, in Primary Health Care and in Hospitals and Conclusions. The Implementation of Case Management Models improves the response to victims of violence, with consequences on the quality of care provided, with an increase in prevention of health results. No studies were found relating the nurse who is the case manager for domestic violence in Primary Health Care.

Keywords: Nursing; Case Management; Domestic Violence; Primary Health Care; Revision.

\section{Introdução}


A Violência Doméstica é uma problemática que preocupa toda a sociedade, tendo um impacto expressivo na saúde e naturalmente na qualidade de vida do indivíduo, família e comunidade, com consequente aumento dos custos em saúde. É um fenómeno multifactorial definido pela Organização Mundial de Saúde (OMS) (Taft et al., 2012) como o uso de força física ou poder contra si mesmo, ou contra outra pessoa, grupo ou comunidade e que tem como consequência a possibilidade de aparecimento de lesões físicas, danos psicológicos, alterações de desenvolvimento, abandono ou negligência e inclusivamente a morte.

A Organização para a Cooperação e Desenvolvimento Económico (OCDE) refere que o principal objetivo de um sistema de saúde é promover a saúde dos utentes e das populações. Ser saudável é também promover o bem-estar pessoal, social e económico, pessoas saudáveis geram comunidades saudáveis e colaboram para uma sociedade mais produtiva, próspera e ativa, uma prática de atendimento positiva é um forte sinal de prestação de cuidados de qualidade (OECD - Organisation for Economic Co-operation and Development, 2019).

Perante esta preocupação e o escasso conhecimento e evidência científica sobre a intervenção do enfermeiro gestor de caso, em situações de Violência Doméstica (VD), em contexto de Cuidados de Saúde Primários (CSP), é indispensável e um dever promover a investigação em enfermagem sobre a temática, sobretudo pelo facto de os estudos publicados serem escassos.Emerge assim a necessidade de investigar a intervenção do enfermeiro gestor de caso a outros níveis de cuidados dos sistemas de saúde, para além do contexto hospitalar.

Reconhecendo o gestor de caso como um coordenador de intervenção de cuidados integrados e complexos, com uma contribuição única nos cuidados sociais e de saúde assim como na participação e na autonomia dos indivíduos em situações complexas de saúde (Lukersmith et al., 2016), parece pertinente a compreensão da importância da intervenção do enfermeiro gestor de caso, ou seja, gestor clínico, em Cuidados de Saúde Primários, em situação de violência doméstica.

Este estudo pretende dar a conhecer o estado da arte, através da utilização deste método de revisão da literatura com PBE. Para tal foi traçado o Objetivo de examinar a evidência científica acerca do enfermeiro gestor de caso, para a prevenção da violência doméstica em CSP.

\section{Métodos}

Esta revisão teve como referência a metodologia proposta por Whittemore \& Knafl (2005) para revisões integrativas: tendo como objetivo "Identificar a evidência científica sobre a intervenção do enfermeiro gestor de caso, na promoção da prevenção da Violência Doméstica". A questão de revisão é: Qual a intervenção do enfermeiro gestor de caso na promoção da prevenção da violência doméstica?

Definiu-se um limite temporal alargado para abranger toda a literatura que abordasse a temática em questão, devido à sua escassez no contexto do papel do Enfermeiro gestor de caso em situação de Violência Doméstica, em Cuidados de Saúde Primários. Assim, os artigos obtidos foram limitados ao período de 2010 a 2020. Esta revisão considerou estudos publicados em inglês, espanhol e português.

Elegeu-se a plataforma EBSCOhost e selecionaram-se as bases bibliográficas eletrónicas: CINAHL, MEDLINE, Cochrane Database. 
Foram utilizados os descritores validados através dos Descritores em Ciências da Saúde - DeCS (compatível com Medical Subject Headings - MeSH): Violência Doméstica (Domestic Violence), Cuidados de Saúde Primários (Primary Health Care), Administração de Caso (Case Management), Saúde Pública (Public Health), Enfermagem (Nursing). Estes foram combinados através das expressões booleanas AND da seguinte forma: Domestic Violence AND Primary Health Care AND Nursing e uma segunda pesquisa utilizando Domestic Violence AND Case Management AND Nursing.

Os artigos disponíveis em texto completo foram considerados para os estudos que continham os critérios de inclusão, ou seja, artigos indexados nos últimos 10 anos que enquadrassem a temática da prevenção da Violência Doméstica em Cuidados de Saúde Primários e o papel de Gestor de Caso em enfermagem, de igual forma enquadrado na temática da violência doméstica.

A seleção dos estudos foi realizada por meio da leitura minuciosa dos títulos e resumos. Desta forma foram apenas considerados os estudos que atendiam aos critérios de inclusão supracitados. Posteriormente dos artigos tidos em consideração, foi realizada a leitura integra do trabalho. Os dados foram extraídos dos artigos incluídos na revisão sistemática, recorrendo-se a uma tabela de extração dos resultados, de acordo com o objetivo e a pergunta da revisão. A tabela de extração foi organizada pelos seguintes dados: Autor(es), Ano de publicação, País; Título; Objetivos; Desenho do estudo; População em estudo/Tamanho da amostra/Participantes; Contexto; Conceito(s) relevantes da questão de revisão; Principais conclusões. Objetivo da investigação, resultados encontrados e implicação para a gestão em enfermagem.

Assim a Figura 1 infra especifica os resultados das etapas de análise, seguindo o modelo PRISMA Flow Diagram (Moher et al, 2009).

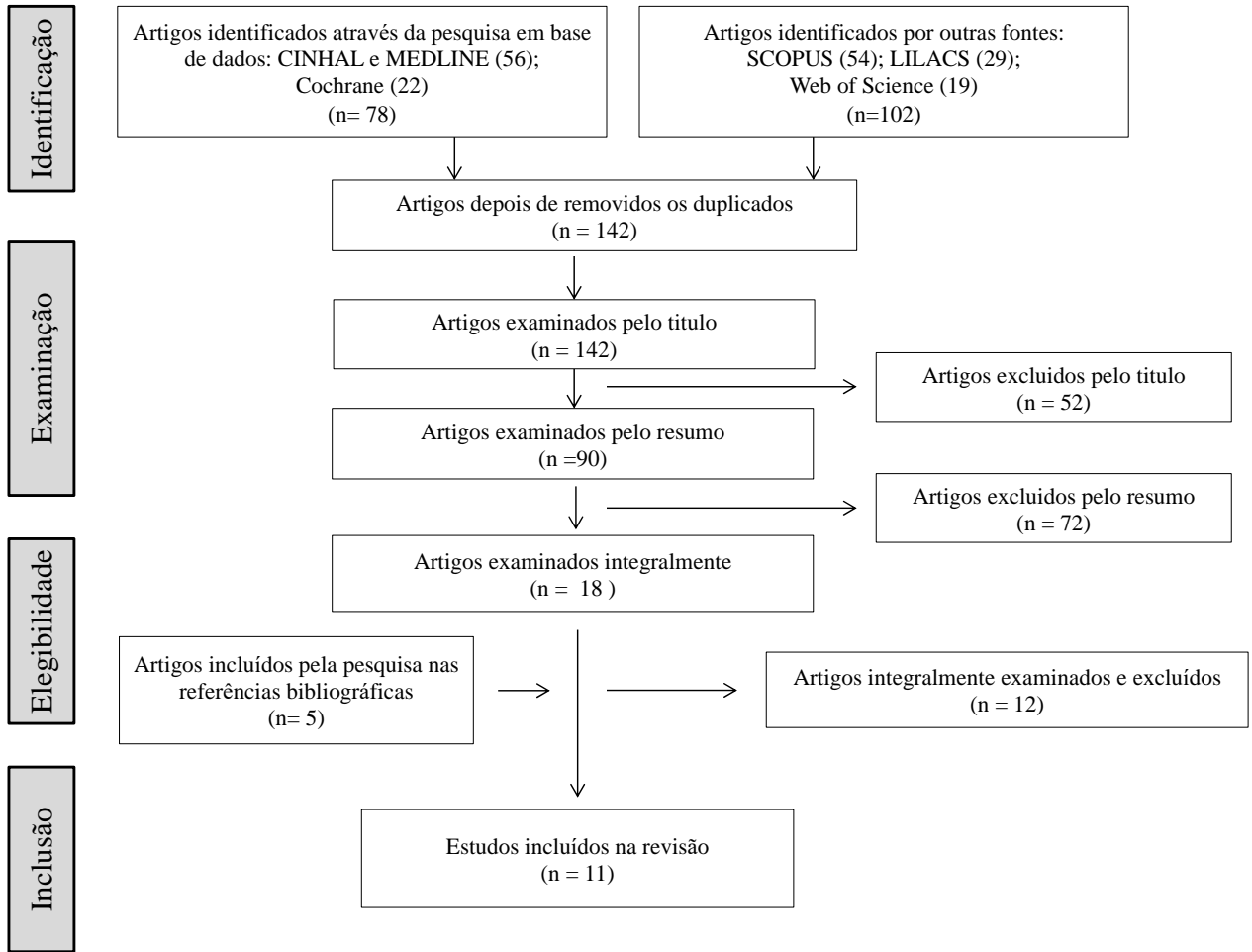

Fig. 1. Diagrama PRISMA referente ao processo de seleção dos estudos. 
Após terem sido retirados os artigos duplicados, foram identificados 142 artigos para seleção da revisão. Um total de 23 artigos cumpria os critérios de inclusão com base na verificação dos títulos e dos resumos, sendo assim selecionadas para o estudo. Uma vez obtidos os artigos de texto integral, estes foram lidos e examinados, de modo a obter a amostra final de 11 artigos que cumpriram os critérios de inclusão, dos quais 4 exclusivamente em contexto da CSP, 1 em contexto hospitalar e 6 em contexto misto, ou seja, todos os contextos de prestação de cuidados CSP e hospitalar, e ONG). O mais recente foi publicado no ano de 2019 e o mais antigo no ano de 2012, sendo um de 2013, um de 2014, três de 2015, dois de 2016 e dois de 2017. Os Estados Unidos da América com o maior número de publicações, quatro publicações sobre a temática, e pelo Brasil com uma publicação, enquanto que a Europa apresentou também quatro publicações, sendo três do Reino Unido e uma de Espanha. Dois estudos, são Australianos.

Pela análise dos resultados dos 11 artigos, surgiram cinco categorias temáticas: O Gestor de caso em situação de VD e a responsabilidade multifacetada; A contribuição do administrador de caso para situações de saúde complexas; A complexa abordagem em contexto de CSP da temática da VD; Modelos de abordagem do fenómeno da VD para a prevenção da mesma e diminuição de complicações; e ainda programas e projetos inovadores, com equipas interdisciplinares para a prestação de cuidados de qualidade a utentes com situações de saúde complexas.

Foram encontradas duas Revisões Sistemáticas da Literatura publicadas em 2016 e 2017 uma que estudou a temática do administrador de casos e sua contribuição para a integração de cuidados aplicados aos CSP, enquanto que a outra pesquisou os variados programas de assistência em saúde para vítimas de violência doméstica, respetivamente.

Embora tenha sido importante incluir revisões sistemáticas, apenas duas incidiam no contexto de cuidados desta revisão, que são metassínteses. A de Sprague et al. (2017) que analisou um total de 43 estudos que consideraram o fenómeno da Violência Doméstica como complexo e um importante problema de Saúde Pública, explicando a diversidade e heterogeneidade do mesmo quanto ao tipo de assistência, como ela é prestada, ou como os próprios estudos são avaliados, que nos criam dúvidas e desafios sobre a perceção de quais são realmente eficazes (Sprague et al., 2017). A revisão de Lukersmith (2016), analisa os fatores relacionados com a gestão de casos, com todas as suas diferentes denominações, variações e contextos, continuando a sustentar a coordenação, integração e gestão dos cuidados e assistência social em muitos contextos diferentes, para diferentes condições de saúde, em contexto de CSP (Lukersmith et al., 2016). Estas revisões de literatura examinaram a evidência científica com objetivos distintos, mas nenhuma versa objectivamente sobre o tema da presente revisão. Assim, considera-se de grande abrangência mundial a evidência nela descrita.

\section{Discussão de Resultados}

\subsection{O Gestor de Caso em Situação de Violência Doméstica e a Responsabilidade Multifacetada:}

O Gestor de caso tem responsabilidades na promoção da prevenção da Violência Doméstica. Para além de se manter atualizado sobre as questões que influênciam a sua prática profissional também deve ter sempre presentes os seus deveres jurídicos e profissionais, ou seja, o gestor de caso pode fazer a diferença ao ponto de salvar uma vida através dos registos e da notificação das situações de risco/perigo (Silva et al., 2017), tendo também um importante papel na capacitação da comunidade para implementar estratégias para interromper a perpetração da VD (Muller, 2014). 


\subsection{A Contribuição do Administrador de Caso para Situações de Saúde Complexas:}

A gestão de casos proporciona uma contribuição única para a integração dos cuidados de saúde, serviços sociais e outros serviços no setor dos CSP para pessoas com condições de saúde complexas (Lukersmith et al., 2016). Também os enfermeiros como gestores de caso "advogam" os utentes, contribuindo também para a economia de custos e outros benefícios que ajudam a sustentar os sistemas de saúde (Armold, 2019).

\subsection{A Complexa Abordagem em Contexto dos CSP da Temática da VD:}

A Violência doméstica é descrita como um grave problema de saúde pública, sendo que as enfermeiras desempenham um papel importante no reconhecimento e na resposta ao fenómeno(Bradbury-Jones, 2015). Muitas têm conhecimento sobre violência doméstica, mas não têm segurança suficiente para questionar os utentes, clientes e alunos/colegas, por forma a identificar as situações(Bradbury-Jones, 2015). Os "Visitantes de saúde", que vivem e trabalham com e para a comunidade, desempenham um papel importante no início de conversas determinantes sobre assuntos domésticos e violência doméstica, podendo utilizar as suas competências relacionais para melhorar os resultados de saúde pública tanto para as crianças, como para as famílias (Bradbury-Jones, 2015).

\subsection{Modelos de Abordagem do Fenómeno da VD para a Prevenção da Mesma e Diminuição de Complicações:}

O artigo de Prieto et al (2015) selecionado para esta revisão integrativa utilizou variados instrumentos de medida e avaliação da VD entre os quais o questionário AAS, "Abuse Assessment Screen", que é usado como instrumento de triagem para avaliação de situações de risco/perigo de violência doméstica; em caso de confirmação de um caso positivo de VD, existe a obrigação legal de completar o registo e a documentação com uma parte específica das lesões. Outra forma de realizar a triagem do fenómeno da VD por forma a analisar o risco é usando o questionário DA "Danger Assessment" (Prieto $S$ et al., 2015).

Também o programa MOVE foi um ensaio clínico randomizado de um modelo aperfeiçoado de triagem de Violência Doméstica e cuidados de suporte por parte de enfermeiras de Saúde Materna e Infantil. O NPT foi desenvolvido a partir do Modelo de Processo de Normalização, é uma teoria de implementação social que fornece uma construção conceptual para compreender o planeamento social do trabalho (implementação), de tornar os procedimentos como elementos habituais da vida quotidiana (incorporação) e de conservar práticas integradas nos seus contextos sociais (integração) O NPT reconheceu a introdução de uma nova prática clínica como um processo social (Hooker \& Taft, 2016).

Ainda foi descrito outro programa - o SMSB - que conquistou ganhos no tratamento do complexo problema da VD durante a gravidez. Estes ganhos refletem o impacto do modelo de prestação de cuidados colaborativos, identificando particularmente como o modelo sustenta a integração clínica e promove sinergias entre os recursos restritos e conjuntos de competências do sistema de saúde e da "agência comunitária" de violência doméstica (Kramer et al., 2012). Os resultados positivos relacionados com saúde e segurança para mulheres e seus bebés são alcançados, assim como os membros das equipas das organizações de saúde e violência doméstica beneficiam também dos serviços de consultoria e educacionais do programa. (Kramer et al., 2012). 
Do mesmo modo foi encontrada descrição de um projeto de melhoria de qualidade sobre a criação, implementação e avaliação de um programa estruturado para detectar e gerir situações de VD num serviço especializado em medicina de emergência (Basu \& Ratcliffe, 2014).

Reconhece-se que uma forte liderança e a priorização do tema dentro do serviço têm facilitado o desenvolvimento dos procedimentos e contribuído substancialmente para o seu sucesso. $\mathrm{O}$ trabalho em rede, realizado com os parceiros da comunidade, tem sido inestimável na adaptação do programa de serviço e educação às necessidades dos funcionários e utentes (Basu \& Ratcliffe, 2014).

\subsection{Programas e Projetos Inovadores, com Equipas Interdisciplinares para a Prestação de Cuidados de Qualidade a Utentes com Situações de Saúde Complexas:}

Para Sprague et al (2017) a maioria dos diferentes tipos de programas de assistência a mulheres em situações de saúde complexas, vítimas de Violência Doméstica, apresentam resultados positivos e foi considerada como benéfica para as mesmas.

Um dos projetos inovadores é descrito como tendo equipas de médicos e enfermeiros que realizam visitas aos utentes ou cuidados de saúde no domicílio, sendo que as equipas também incluem assistentes sociais, fisioterapeutas ou até capelões(Davis, 2015). Para Davis (2015) a instalação de modelos de atendimento inovadores, envolve todo um sistema, não apenas uma linha de serviço ou uma especialidade. A instalação requer interrupção, com um contexto cultural para apoiá-la. Uma cultura dentro da qual a rutura pode ocorrer é aquela construída sobre alinhamento, visão compartilhada, propósito compartilhado, comunicação, campeões identificados e continuidade do cuidado.

\subsection{Limitações do Estudo}

Devido à especificidade do tema do enfermeiro gestor de caso e do contexto da prevenção da Violência Doméstica em CSP, bem como à respectiva escassez de evidência científica, uma limitação deste estudo foi apenas existirem três artigos dos últimos três anos do período de pesquisa. Em relação aos últimos cinco anos do período temporal da pesquisa, os estudos incluídos foram apenas cinco, do total dos onze analisados nesta revisão.

\subsection{Contribuições para a Área da Enfermagem}

Existe um reduzido conhecimento sobre o enfermeiro gestor de caso para a Prevenção da Violência doméstica nos CSP. Esta revisão de literatura pretende contribuir para aumentar esse conhecimento nas diferentes áreas de atuação dos enfermeiros: na prestação de cuidados, na gestão, na investigação, na docência e na consultoria ou assessoria.

\section{Conclusões}

Esta revisão integrativa fornece alguma evidência sobre o enfermeiro gestor de caso em situação de VD em contexto de CSP e presta um serviço aos enfermeiros, aos enfermeiros gestores, aos académicos, aos investigadores e aos responsáveis pela definição de políticas de saúde, apresentando contributos para a melhoria da qualidade dos cuidados de saúde e particularmente de enfermagem, a pessoas em situação de VD, em contexto dos CSP. São aspectos importantes a considerar em contexto de CSP, a relação multidisciplinar entre enfermeiros e médicos, assim como entre enfermeiros, gestores e administradores. 
Também é relevante a consideração de uma boa comunicação nas Unidades de Saúde e entre as organizações, por forma a realizar um trabalho em rede de prevenção do fenómeno da Violência Doméstica.

O fenómeno de VD é complexo e deve ser compreendido e trabalhado em contexto de CSP em interligação com o contexto hospitalar, pelo tipo de tomada de decisão, relação entre os membros das equipas e pelos processos organizacionais.

Também é necessário promover a sistematização e a triagem das situações de VD que vão contribuir para o aumento dos resultados com os clientes, enfermeiros e organizações, ou seja, com todos os atores envolvidos. Para atingir essa finalidade apresenta-se como mais-valia o enfermeiro gestor de caso, que pelas suas competências e características profissionais pode contribuir também para a economia de custos e outros benefícios que ajudam a sustentar os sistemas de saúde.

\section{Referências}

Amestoy, S. C. oelh., Backes, V. M. S. chuber., Thofehrn, M. B. us., Martini, J. G. u., Meirelles, B. H. örne. S., \& Trindade, L. de L. (2014). [Conflict management: challenges experienced by nurse-leaders in the hospital environment]. Revista Gaúcha de Enfermagem / EENFUFRGS, 35(2), 79-85. https://doi.org/10.1590/1983-1447.2014.02.40155

Armold, S. (2019). Case management: An overview for nurses. Nursing, 49(9), 43-45. https://doi.org/10.1097/01.NURSE.0000577708.49429.83

Basu, S., \& Ratcliffe, G. (2014). Developing a multidisciplinary approach within the ED towards domestic violence presentations. Emergency Medicine Journal, 31(3), 192-195. https://doi.org/10.1136/emermed-2012-201947

Bradbury-Jones, C. (2015). Talking about domestic abuse: Crucial conversations for health visitors. Community Practitioner, 88(12), 40-43.

Davis, K. (2015). Innovative Models of Care Delivery: Addressing Transitions Across The Care Continuum. In Innovative Models of Care Delivery: Addressing Transitions Across The Care Continuum (Issue Hospital at Home®: Inovation in Practice, pp. 17-20).

Hooker, L., \& Taft, A. (2016). Using theory to design, implement and evaluate sustained nurse domestic violence screening and supportive care. Journal of Research in Nursing, 21(5/6), 432-442. https://doi.org/10.1177/1744987116649633

Kramer, A., Nosbusch, J. M., \& Rice, J. (2012). Safe mom, safe baby: A collaborative model of care for pregnant women experiencing intimate partner violence. Journal of Perinatal and Neonatal Nursing, 26(4), 307-316. https://doi.org/10.1097/JPN.0b013e31824356dd

Lukersmith, S., Millington, M., \& Salvador-Carulla, L. (2016). What is case management? A scoping and mapping review. International Journal of Integrated Care, 16(4), 1-13. https://doi.org/10.5334/ijic.2477

Moher D, Liberati A, Tetzlaff J, Altman DG, The PRISMA Group (2009). Preferred Reporting Items for Systematic Reviews and Meta-Analyses: The PRISMA Statement. PLoS Med 6(7): e1000097. https://doi.org/10.1371/journal.pmed.1000097

Moher D, Liberati A, Tetzlaff J, Altman DG, The PRISMA Group (2009). Preferred Reporting Items for Systematic Reviews and Meta-Analyses: The PRISMA Statement. . Annals of Internal Medicine, 14 https://www.acpjournals.org/doi/10.7326/0003-4819-151-4-200908180-00135

Muller, L. S. (2014). A case management briefing on domestic violence. Professional Case Management, 19(5), 237-240. https://doi.org/10.1097/NCM.0000000000000054

OECD - Organisation for Economic Co-operation and Development. (2019, November 7). Health at a Glance 2019. OECD. https://doi.org/10.1787/4dd50c09-en

Prieto S, G., Buendía E, L., Corbalán M C, M., \& Mogente A, C. (2015). Cribado de violencia de género en un departamento de salud. Revista Investigacion \& Cuidados, 13(33), 6-12. 
Silva, N. N. F., Leal, S. M. C., Trentin, D., Vargas, M. A. D. O., Vargas, C. P., \& Vieira, L. B. (2017). Atuação Dos Enfermeiros Da Atenção Básica a Mulheres Em Situação De Violência. Enfermagem Em Foco, 8(3), 70-74. https://doi.org/10.21675/2357-707x.2017.v8.n3.1290

Souza, M. T. de, Silva, M. D. da, \& Carvalho, R. de. (2010). Revisão integrativa: o que é e como fazer. Reme: Revista Mineira de Enfermagem, 8(1), 102-106. https://doi.org/10.1590/S167945082010RW1134

Sprague, S., Scott, T., Garibaldi, A., Bzovsky, S., Slobogean, G. P., McKay, P., Spurr, H., Arseneau, E., Memon, M., Bhandari, M., \& Swaminathan, A. (2017). A scoping review of intimate partner violence assistance programmes within health care settings. European Journal of Psychotraumatology, 8(1), 13. https://doi.org/10.1080/20008198.2017.1314159

Taft, A. J., Small, R., Humphreys, C., Hegarty, K., Walter, R., Adams, C., \& Agius, P. (2012). Enhanced maternal and child health nurse care for women experiencing intimate partner/family violence: protocol for MOVE, a cluster randomised trial of screening and referral in primary health care. BMC Public Health, 12, 811. https://doi.org/10.1186/14712458-12-811

Whittemore, R. \& Knafl, K. (2005). The integrative review: updated methodology. Journal of Advanced Nursing, 52(5), 546-553. https://doi.org/10.1111/j.1365-2648.2005.03621.x 